\title{
Introduction to the focused section on intelligent robotics for rehabilitation and human assistance
}

\author{
Jingang $\mathrm{Yi}^{1}{ }^{1} \cdot$ Jun Ueda $^{2} \cdot$ Xiangyang $\mathrm{Zhu}^{3}$
}

Received: 13 December 2016/ Accepted: 28 December 2016/Published online: 10 January 2017

(C) Springer Singapore 2017

The recent years witness an increasing demand for rehabilitation and human assistive devices that maximize the effectiveness/efficiency of the clinical therapy and develop innovative solutions to promote independent living of senior citizens as well as persons. Intelligent robotics plays a central role in the development of these rehabilitation and human assistive devices. Moreover, recent advances in computational intelligence, and sensing and control technologies enable new applications in human motion analysis, diagnosis, monitoring and feedback allowing more autonomous personalized treatments without the need for constant therapist interaction.

In an effort to disseminate current advances and identify challenges and opportunities, this "Focused Section on Intelligent Robotics for Rehabilitation and Human Assistance" of the International Journal of Intelligent Robotics and Applications (IJIRA) highlights several important technology achievements in design, modeling and control of rehabilitation and human assistive robotic systems. The Focused Section includes six papers out of seventeen submissions that represent a sample of current developments of intelligent robotics for rehabilitation and human assistance.

Jingang Yi

jgyi@rutgers.edu

1 Department of Mechanical and Aerospace Engineering, Rutgers, The State University of New Jersey, Piscataway, NJ 08854-8058, USA

2 George W. Woodruff School of Mechanical Engineering, Georgia Institute of Technology, Atlanta, GA 30332-0405, USA

3 School of Mechanical Engineering, Shanghai Jiaotong University, Shanghai 200240, People's Republic of China
The paper "Bilateral control of functional electrical stimulation and robotics-based tele-rehabilitation" from Alibeji et al. presents a position-synchronization control system for functional electrical stimulation (FES)-based tele-rehabilitation to provide remotely controlled physical assistance. The proposed controller synchronizes an FESdriven human limb with a remote physical therapist's robotic manipulator despite constant bilateral communication delays. The controller overcomes a major stability analysis challenge under the unknown and unstructured nonlinearities in the FES-driven musculoskeletal dynamics through neural network models and an adaptation design. The performance of the state synchronization controller is validated through demonstrated bilateral control of FESelicited leg extension and a human-operated robotic manipulator.

The second paper "Estimating the multivariable human ankle impedance in dorsi-plantarflexion and inversion-eversion directions using EMG signals and artificial neural networks" from Dallali et al. describes a new modeling framework for the relationship between the ankle impedance and lower extremity muscle activations. Such relationship is crucial for control and design of ankle-foot prosthesis for transtibial amputees. The authors propose to use artificial neural networks (ANN) to characterize the relationship between the lower extremity muscle EMG signals and the ankle impedance in the sagittal and frontal planes. Anklebot, a highly back-drivable, safe, and therapeutic robot is used to apply stochastic position perturbations to the human ankle in dorsi-plantar-flextion (DP) and inversion-eversion (IE) directions. The promising results of this study show that a similar approach can be used during human dynamic walking.

The work from Zhang and Ye is on "RGB-D camera based walking pattern recognition by support vector 
machines for a smart rollator." This paper presents a walking pattern detection method for a smart rollator by using the depth data of an RGB-D camera for the user's lower extremities. The method segments the $3 \mathrm{D}$ point data of the lower extremities into the leg and foot data points, from which a skeletal system with six skeletal points and four rods is extracted and used to represent a human walking gait. A gait feature, comprising the parameters of the gait shape and gait motion, is then constructed to describe a walking state. Using these key gait features, a walking video sequence is modeled as a Markov chain and three Support Vector Machines (SVMs) are trained for walking pattern detection. Experimental results demonstrate that the method has a better performance in detecting walking patterns than seven other existing approaches.

In "Robotic assistance for children with cerebral palsy based on learning from tele-cooperative demonstration" from Najafi et al. a robotic-assistance-as-needed framework is proposed for children with Cerebral Palsy (CP) to perform 2-dimensional (2D) position following task through tele-manipulating the slave robot in the play environment. Using a learning-fromdemonstration strategy, the Gaussian Mixture Model (GMM) and Gaussian Mixture Regression (GMR) techniques are used to approximate the average and variability of the demonstrated therapist-child trajectories. The robotic system uses the impedance/admittance control design in the robotic assistance phase (without therapist intervention) such that the master robot provides a desirable flexibility for the child to follow the average trajectory.

The work presented in "Influence of rolling resistance on manual wheelchair dynamics and mechanical efficiency" from Teran and Ueda discuss several aspects of the wheelchair dynamics and control. The paper presents two controller designs for anatomical model propulsion system (AMPS) for wheelchairs to enhance motion repeatability and precision in outdoor environment. The paper also presents an estimation method to accurately predict rolling assistance and mechanical efficiency, in the form of cost of transport (COT), under various motion conditions. One of the main contributions of the work lies in that the proposed different metrics of mechanical efficiency can potentially become a new standard for comparing performance among different wheelchair models and component configurations. The methodology and metrics presented in this paper could become the cornerstone for the new approach of testing and characterizing manual wheelchairs.

The last paper "Generation of comfortable lifting motion for human transfer assistant robot" from Ding et al. discusses two methods to generate robotic lifting motions to transfer a patient between a bed and a wheelchair, which are used widely in hospitals or nursey homes. The robotic holding posture is generated by using human-robot interactive models and a musculoskeletal model is developed to estimate muscle force and to reduce the interaction forces. To reduce pain, a softness distribution model is created to regulate the interaction force between the robot and the soft human body. The robotic motion for raising the human body from the supine posture to the estimated holding posture is generated by a model-free method with the user's comfortable feedback. Using Bayesian optimization, the number of required physical human-robot interaction trials is reduced to ease the user's burden. The authors have conducted various experiments with a dual arm robotic system and human subjects to validate and evaluate the effectiveness of the design.

We wish to extend our gratitude to all of the authors and anonymous reviewers for their excellent efforts to ensure the quality of the Focused Section papers. We also wish to thank Editor-in-Chief, Professor Kok-Meng Lee, and the additional Associate Editors of the IJIRA for their extraordinary efforts and guidance throughout the production of this Focused Section.

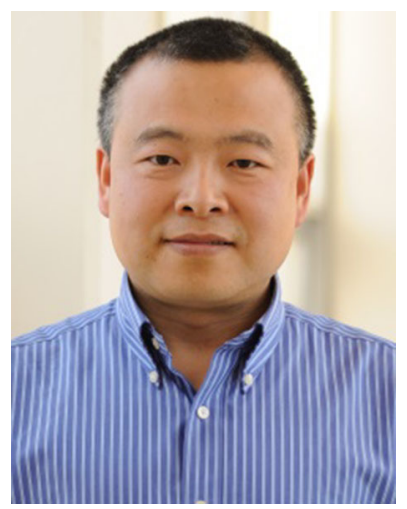

Jingang Yi received the B.S. degree in electrical engineering from Zhejiang University, Hangzhou, China, in 1993, the M.Eng. degree in precision instruments from Tsinghua University, Beijing, China, in 1996, and the M.A. degree in mathematics and the Ph.D. degree in mechanical engineering from the University of California, Berkeley, in 2001 and 2002, respectively. He is currently an Associate Professor of Mechanical Engineering at

Rutgers University. His research interests include autonomous robotic systems, dynamic systems and control, mechatronics, automation science and engineering, with applications to biomedical systems, civil infrastructure and transportation systems. Dr. Yi is a member of American Society of Mechanical Engineers (ASME). He is a recipient of the 2010 US NSF CAREER Award. He has co-authored papers that have been awarded several best papers at IEEE/ASME AIM, ASME DSCC, IEEE ICRA etc. Dr. Yi serves as an Associate Editor for the IEEE Transactions on Automation Science and Engineering, IEEE/ ASME Transactions on Mechatronics, IFAC Journal Mechatronics, IFAC Journal Control Engineering Practice, ASME Journal of Dynamic Systems, Measurement and Control, International Journal of Intelligent Robotics and Applications, and the IEEE Robotics and Automation Society Conference Editorial Board (since 2008). He also served as a Guest Editor for the IEEE Transactions on Automation Science and Engineering in 2009 and an Associate Editor for the ASME Dynamic Systems and Control Division Conference Editorial Board from 2008 to 2010 . 


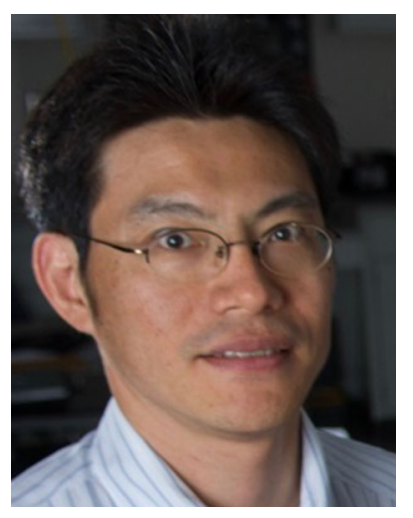

Jun Ueda received the B.S., M.S., and Ph.D. degrees from Kyoto University, Kyoto, Japan, in 1994, 1996, and 2002 all in Mechanical Engineering. From 1996 to 2000, he was a Research Engineer at the Advanced Technology Research and Development Center, Mitsubishi Electric Corporation, Hyogo, Japan. He was an Assistant Professor of Nara Institute of Science and Technology, Japan, from 2002 to 2008. During 2005-2008, he was a visiting scholar and lecturer in the Department of Mechanical Engineering, Massachusetts Institute of Technology. He joined the G. W. Woodruff School of Mechanical Engineering at the Georgia Institute of Technology as an Assistant Professor in 2008 where he is currently an Associate Professor and Woodruff Faculty Fellow. He is also the Director for Robotics PhD Program at Georgia Tech. He received a Fanuc FA Robot Foundation Best Paper Award in 2005, IEEE Robotics and Automation Society Early Academic Career Award in 2009, and Advanced Robotics Best Paper Award in 2015.
Dr. Ueda has published more than 100 peer-reviewed journal papers, conference papers and book chapters. He has issued 13 international and U.S. patents.

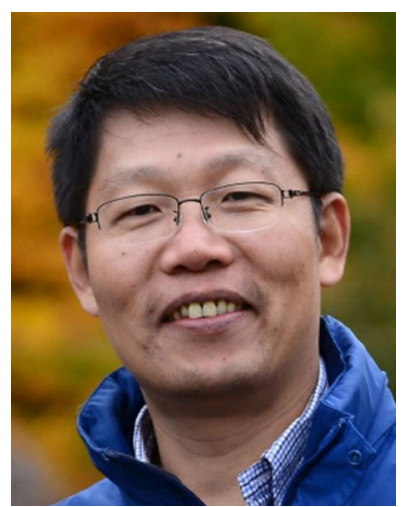

Xiangyang Zhu received his $\mathrm{PhD}$ degree in control engineering from Southeast University, Nanjing China, in 1992. He is currently a Chair Professor of mechatronics and the Director of Robotics Institute at Shanghai Jiao Tong University, Shanghai, China. His research interests include robot manipulation planning, continuum and soft robotics, rehabilitation assistive robotics, neuro-interfacing, and design of dexterous prosthetic hands. Dr. Zhu has received a number of awards including the National Science Fund for Distinguished Young Scholars from NSFC in 2005, and the Changjiang Distinguished Professorship from the Ministry of Education in 2007. He is currently an Associate Editor of IEEE Transactions on Cybernetics and Journal of Bionic Engineering. 